\title{
Cardiovascular disease Detection through Wearable Home Care Assisted Living System
}

\author{
Pravin Wararkar \\ Department of \\ Electronics \& Telecommunication \\ Engineering (Assistant Professor) \\ SVKM's NMIMS (Deemed-to-be-University) \\ Mukesh Patel School of Technology Management \\ \& Engineering. Shirpur \\ Shirpur , India
}

\author{
Aastha Gupta \\ Department of \\ Information Technology (Student) \\ SVKM's NMIMS (Deemed-to-be-University) \\ Mukesh Patel School of Technology Management \\ \& Engineering. Shirpur \\ Shirpur, India
}

\author{
Ankit Dixit \\ Department of \\ Electronics \& Telecommunication \\ Engineering (Assistant Professor) \\ SVKM's NMIMS (Deemed-to-be-University) \\ Mukesh Patel School of Technology Management \\ \& Engineering. Shirpur \\ Shirpur , India
}

\author{
Samay Mehta \\ Department of \\ Information Technology (Student) \\ SVKM's NMIMS (Deemed-to-be-University) \\ Mukesh Patel School of Technology Management \\ \& Engineering. Shirpur \\ Shirpur , India
}

\begin{abstract}
The significance of developing an Assisted Living System (AALT) is increasing with the development in technology and an upward drift in independent living. The need is to develop a robust and accurate system which monitors the health of people, concentrating on elderly people. In this research work, the idea of wearing a Bluetooth integrated heart rate monitor wrist band to record and analyse the daily activities of the personnel has been proposed. The main concern is to provide them an environment which does not restrict their daily activities, understanding the human psychology. The hypothesis that wearable band can be used to examine the target heart rate and average maximum heart rate for detecting the health issue is being evaluated. However, the implementation of the same system in practical needs future research.
\end{abstract}

\section{General Terms}

Your general terms must be any term which can be used for general classification of the submitted material such as Pattern Recognition, Security, Algorithms et. al.

\section{Keywords}

Target heart rate, resting heart rate, AALT

\section{INTRODUCTION}

The Ambience Assisted Living Technologies (AALT) which are developed for countering the effects of elderly growing population to achieve high level of security, interoperability , usability, real- world environment and accuracy needs proper use of welfare technologies. AALT is becoming common area of interest because as with old age disease become more frequent, thus people often require more attention and health care. This demographic trend in the population along with the independent living culture elevates the home health care method of care for the elderly. The prime objective of an AALT system can be divided into the following objectives:
1. To understand the social and political constraints on welfare technology and designing them accordingly.

2. To analyse the need of the users by observation, interaction or through participatory design with the potential stakeholders.

3. To participate with end users to identify the criteria's of evaluation for the system.

4. To recommend different welfare technologies along with the end user participation.

In the recent year's variety of devices have been developed such as motion sensors, radio-frequency devices, cameras to monitor elders. But the fundamental problem with all such devices, specifically regarding wearable cameras or stationary cameras is that it restricts the natural response of the end users.

Example solution of motion sensors was proposed by authors of [3] by the use of 3 axis-accelerometers which consists of $5 \mathrm{~g}$ to $+5 \mathrm{~g}$ accelerometers mounted on a single block, by using the appropriate data collection of both hardware and software will result out in the net acceleration, by computing all the three components. Motion sensors can be integrated into clothing or attached directly on the objects to be monitored, and it is possible that psychological aspects are not recorded using motion sensors.

The example solution of radio-frequency has been proposed by authors of [2] which consist of Wireless Network Systems (WNS), which only use the Received Signal Strength (RSS) for performing task such as device-free localization and the human localization. The system locates the subjects by the signals received from the monitored area. It indicates the movement of doors and also by monitoring the pressure sensors mounted on armchairs, bed which supports detection of sitting or lying postures. 
Among all the solutions the authors of [1] and [4] have proposed the solution using optical observations; they serve to the elders for their independent and safe living using comfortable wearable accessories. The author [1] has used small in size and number of cameras for easy deployment in the environment and easy monitoring, cameras are deployed in different corners of the room to capture images from different angles. On the same platform author [4] has proposed the use of wearable cameras on chest and monitor the surroundings so that the system can deal with privacy and unobtrusive issues more effectively, so as to provide more comfortable invigilation environment. In [1] and [4] authors have studied and formulated how videos can be monitored and processed for effective home care system.

In this research work daily activity analysis has been proposed using wearable band, without the need of recording video and audio data. All the necessarily data analysis should be performed in real-time using efficient mobile application platforms available today. The only information recorded is intermediate data representation and activity heart rate analysis result.

Here, a person's heart rate is mainly considered in indoor environment by heart rate detection. This paper is organized as follows: Section II contains the proposed architecture for assisted living system along with hardware and software propositions. Section III showcases the aim of research work. Finally, Section IV concludes the research work and provides future research directions.

\section{PROPOSED METHODOLOGY FOR ASSISTED LIVING SYSTEM.}

The primary gadget that is being used in the system is an integrated wrist band which has inbuilt heart rate sensor. It has its inbuilt Bluetooth module, the collected data is then sent to the mobile device through Bluetooth transfer on which the further processing is done by the application developed and deployed is platform independent after the data has

been collected and processed it is sent to the local server and will further send the status of the monitored person to the prescribed doctor, on his/her application service as shown in Fig 1 .

The Bluetooth integrated heart monitor, capture minute changes in the health of the person, which is not captured appropriately in the existing home care systems which includes wearable cameras or stationary cameras, or radio frequency based systems.

The use of integrated band allows detection and monitoring option in various situations and actions that can be difficult to detect using wearable or stationary cameras or radio frequency equipments. For example, it is convenient and

possible to monitor events related to close field detection, like having normal workout session, interacting with others, or performing other activities.

\subsection{Hardware propositions}

Processing heart rate from the wearable integrated band in real-time is computationally complex task. Thus, it becomes important to make an appropriate choice of embedded hardware and selection of fast and accurate algorithm that covers different scenarios.

In order to achieve required processing speed, specialized hardware can be used. On the other hand, in a typical household, there are many different devices (i.e. mobile phones, tablets and smart TVs). The main purpose is to build a system which is affordable by maximum people. Here Bluetooth integrated wrist bands such as Suunto Smart Sensor or Jawbone UP3 and UP4 are being used. And the application software will be built on both Android operating system platform and IOS.

\subsection{Algorithm Propositions}

The algorithm of the system mainly depends on the various heart rate limits which vary for males and females depending on the age factor.

\section{Maximum and target heart rate}

There is no definite limit, when the resting rate is high, but it has been observed that consistent heart rate in the upper level, can be much stressful for heart and other organs. If a person has a high heart rate at rest and is experiencing other symptoms, then it is required to have a heart check up. The resting heart rate categorized for men and women, along on the basis of their age is shown In TABLE I and TABLE II.

Table 1. Resting heart rate for women

\begin{tabular}{|l|c|c|c|c|c|c|}
\hline \multicolumn{1}{|c|}{ Age } & $\mathbf{1 8 - 2 5}$ & $\mathbf{2 6 - 3 5}$ & $\mathbf{3 6 - 4 5}$ & $\mathbf{4 6 - 5 5}$ & $\mathbf{5 6 - 6 5}$ & $\mathbf{6 5 +}$ \\
\hline Athlete & $54-60$ & $54-59$ & $54-59$ & $54-60$ & $54-59$ & $54-59$ \\
\hline Excellent & $61-65$ & $60-64$ & $60-64$ & $61-65$ & $60-64$ & $60-64$ \\
\hline Good & $66-69$ & $65-68$ & $65-69$ & $66-69$ & $65-68$ & $65-68$ \\
\hline Above & $70-73$ & $69-72$ & $70-73$ & $70-73$ & $69-73$ & $69-72$ \\
Average & & & & & & \\
\hline Average & $74-78$ & $73-76$ & $74-78$ & $74-77$ & $74-77$ & $73-76$ \\
\hline Below & $79-84$ & $77-82$ & $79-84$ & $78-83$ & $78-83$ & $77-84$ \\
Average & & & & & & \\
\hline Poor & $85+$ & $83+$ & $85+$ & $84+$ & $84+$ & $84+$ \\
\hline
\end{tabular}

Table 2. Resting heart rate for men

\begin{tabular}{|l|c|c|c|c|c|c|}
\hline \multicolumn{1}{|c|}{ Age } & $\mathbf{1 8 - 2 5}$ & $\mathbf{2 6 - 3 5}$ & $\mathbf{3 6 - 4 5}$ & $\mathbf{4 6 - 5 5}$ & $\mathbf{5 6 - 6 5}$ & $\mathbf{6 5 +}$ \\
\hline Athlete & $49-55$ & $49-54$ & $50-56$ & $50-57$ & $51-56$ & $50-55$ \\
\hline Excellent & $56-61$ & $55-61$ & $57-62$ & $58-63$ & $57-61$ & $56-61$ \\
\hline Good & $62-65$ & $62-65$ & $63-66$ & $64-67$ & $62-67$ & $62-65$ \\
\hline $\begin{array}{l}\text { Above } \\
\text { Average }\end{array}$ & $66-69$ & $66-70$ & $67-70$ & $68-71$ & $68-71$ & $66-69$ \\
\hline Average & $70-73$ & $71-74$ & $71-75$ & $72-76$ & $72-75$ & $70-73$ \\
\hline $\begin{array}{l}\text { Below } \\
\text { Average }\end{array}$ & $74-81$ & $75-81$ & $76-82$ & $77-83$ & $76-81$ & $74-79$ \\
\hline \begin{tabular}{l} 
Poor \\
\hline
\end{tabular} & $82+$ & $82+$ & $83+$ & $84+$ & $82+$ & $80+$ \\
\hline
\end{tabular}


When people exercise in their "target heart zone," they gain the most benefits and improve their heart's health. When the heart rate is in the target zone, people know that muscles are being pushed to get stronger.

A person's target heart rate zone is between 50 -85 percent of his or her maximum heart rate. Maximum heart rate is calculated by simply subtracting age from 220: 220 - Age. For a 35-year-old person, for example:

\section{$220-35=185$}

The target zone for a 40-year-old person would be between 50 and 85 percent of his or her maximum heart rate:

- $\quad 50$ percent level: 185 x $0.50=92.5 \mathrm{bpm}$

- 85 percent level: 185 x $0.85=157.25$ bpm

Table 3. Table captions should be placed above the table

\begin{tabular}{|c|c|}
\hline $\begin{array}{c}\text { Age } \\
\text { (in years) }\end{array}$ & $\begin{array}{l}\text { Average Maximum Heart } \\
\text { Rate, 100\% }\end{array}$ \\
\hline 20 & $193 \mathrm{bpm}$ \\
\hline 30 & $190 \mathrm{bpm}$ \\
\hline 35 & $185 \mathrm{bpm}$ \\
\hline 40 & $180 \mathrm{bpm}$ \\
\hline
\end{tabular}

\begin{tabular}{|l|l|}
\hline 45 & $175 \mathrm{bpm}$ \\
\hline 50 & $170 \mathrm{bpm}$ \\
\hline 55 & $165 \mathrm{bpm}$ \\
\hline 60 & $160 \mathrm{bpm}$ \\
\hline 65 & $155 \mathrm{bpm}$ \\
\hline 70 & $150 \mathrm{bpm}$ \\
\hline
\end{tabular}

The formula for maximum heart rate works well for people under 40 but for older people it may overestimate their maximum heart rate. For older people, a better formula for the maximum heart rate is: $208-(0.75 \mathrm{x}$ Age $)$.

The heart rate above the target zone is very dangerous for the individual, thus understanding the maximum target heart rate helps to identify the maximum limit of the heart rate for the person being supervised. Thus after the heart rate is collected the algorithm will check the average maximum heart rate and will send an alert to the doctor on his application interface. The average maximum heart rates are displayed in TABLE III.

\section{WEARABLE PLATFORM}
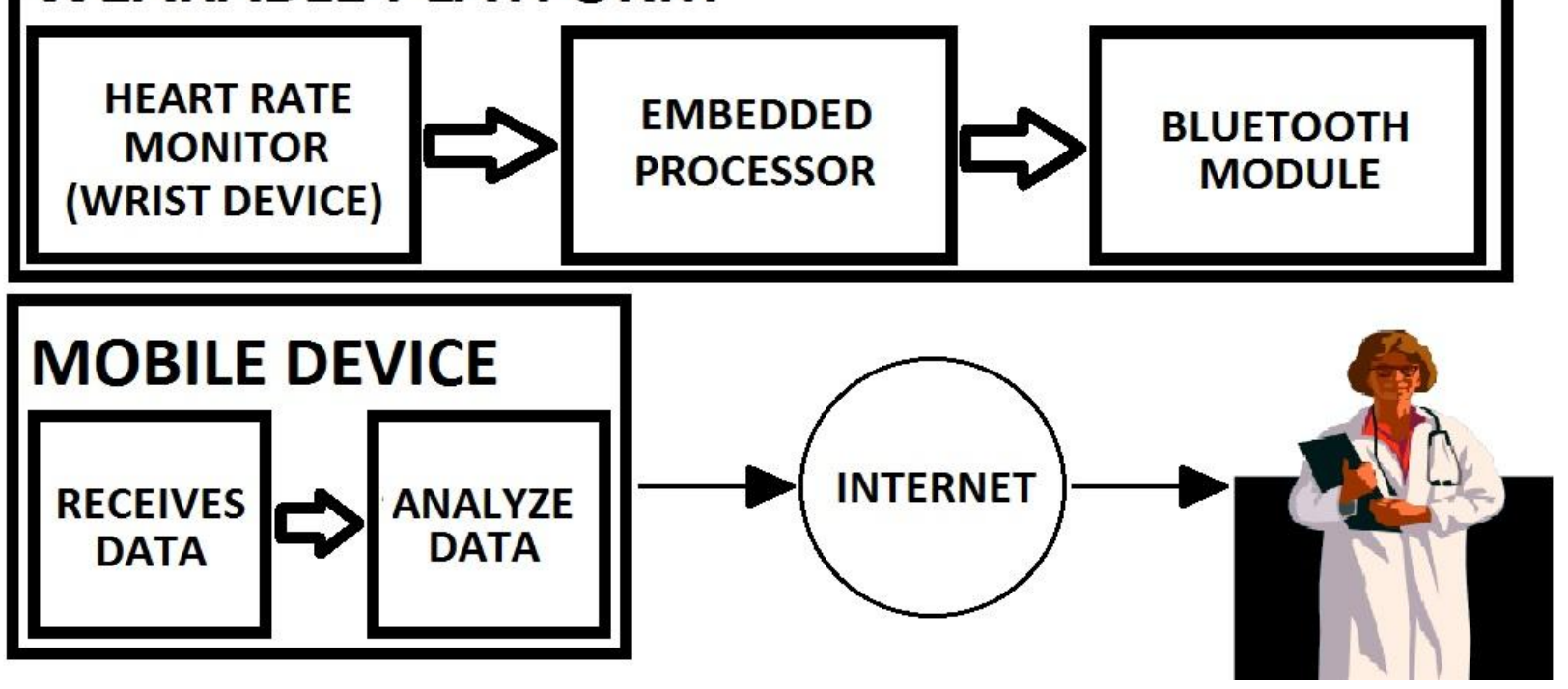

Fig 1: General Overview of Architecture

\section{AIM AND OBJECTIVES OF RESEARCH WORK}

The aim and objectives of research work are:

1. To provide a better living support to the individual living people, by providing them the medication on time.

2. To help people regulate their exercise schedule for maximum benefit.

3. To study the systolic and diastolic figures of individual by their heart rate to provide medication based on blood pressure categorization.

\section{APPLICATIONS}

1. Helping the individual to reach the maximum target heart rate while performing exercises thus gaining the maximum benefit.

2. Indicating the individual that his/her resting heart rate comes into which category and thus suggesting the required exercises.

3. Alerting the doctor when the patient's heart rate is above the average maximum heart rate and providing him the needed medical facilities. 


\section{CONCLUSION}

The detection of any change in the heart rate of the monitored person and thus providing the medical facilities is the key success to cardiovascular disease detection system. The integrated wearable band acts as the primary source of the data collection and finally analyzed by the application logic. Our approach is efficient in capturing different close field, interactions and not hindering the natural response of the invigilated person.

\section{FUTURE SCOPE}

The further research and development of the proposed solution requires attention on the following points:

1. Finding an effective way to calculate systolic and diastolic figures by detecting heart rate of the individual, making the detection of any cardiovascular disease easy.

2. Testing the robustness of the developed method to various conditions, evaluating the hardware components and the algorithm speed optimizations are required.

3. Developing higher level of analysis and heart rate recognition.

\section{REFERENCES}

[1] R. Megret, D. Szolgay, J. Benois-Pineau, P. Joly, J. Pinquier, J.-F. Dartigues, and C. Helmer, "Wearable video monitoring of people with age dementia: Video indexing at the service of healthcare," in Content- Based Multimedia Indexing, 2008. CBMI 2008. International Workshop on, June 2008, pp. 101-108.
[2] Z. Zhou, X. Chen, Y.-C. Chung, Z. He, T. Han, and J. Keller, "Activity analysis, summarization, and visualization for indoor human activity monitoring," Circuits and Systems for Video Technology, IEEE Transactions on, vol. 18, no. 11, pp. 1489-1498, Nov 2008.

[3] A. Khan, Y.-K. Lee, S. Lee, and T.-S. Kim, "Accelerometers position independent physical activity recognition system for long-term activity monitoring in the elderly," Medical \& Biological Engineering \&Computing, vol. 48, no. 12, pp. 1271-1279,2010.

[4] Jaromir Przybylo, "Landmark detection for wearable home care ambient assisted living system", in IEEE ,2010.

[5] Caitlin Hill, Grant Raymond, Irene Yeung, Case Study on "Ambient Assisted Living Technology", submitted on, May 7, 2013.

[6] M.Bocca, S.Gupta, O.Kaltiokallio, B.Mager, Q. Tate, S.Kasera, N. Patwari1, S.Venkatasubramanian."RFbased Device-Free Localization and Tracking for Ambient Assisted Living"

[7] Feng Zhou, Jianxin Jiao, Songlin Chen, Daqing Zhang."A Case-Driven Ambient Intelligence System for Elderly in-Home Assistance Applications", in IEEE,vol.41 ,issue no 2,2011.

[8] Oresko, J.J.; Zhanpeng Jin; Jun Cheng; Shimeng Huang; Yuwen Sun; Duschl, H.; Cheng, A.C."A Wearable Smartphone-Based Platform for RealTime Cardiovascular Disease Detection Via Electrocardiogram Processing", in IEEE,vol.14 ,issue no. 3,2010 . 